\title{
Protocolo de entrenar actores para escenarios de alta fidelidad en educación médica
}

\author{
Protocol of training actors to simulate high fidelity \\ scenarios in medical education \\ Gleyvis Coro-Montanet,* Montserrat Diéguez-Pérez, ${ }^{\ddagger}$ Fátima Cerdán-Gómez, ${ }^{\S}$ \\ María Rosa García-Villalobos," Margarita Gómez-Sánchez," \\ María Jesús Pardo-Monedero**
}

\begin{abstract}
Palabras clave: Aprendizaje basado en simulación, participante simulado, paciente estandarizado, entrenamiento actoral, briefing del actor.

Keywords: Simulation-based learning, simulated participant, standardized patient, actor training, actor briefing.
\end{abstract}

\footnotetext{
* Doctora en Ciencias de la Educación y Doctora en Estomatología. Coordinadora de Simulación Clínica. Profesora adjunta Universidad Europea de Madrid.

‡ Doctor en Odontología. Profesor adjunto

Universidad Europea.

$\S$ Profesora asociada

Universidad Europea.

\| Profesora asociada

Universidad Europea.

" Doctora en

Odontología. Vicedecana de Odontología.

** Doctora en

Medicina. Directora del Departamento de Odontología Preclínica.
}

\section{RESUMEN}

Los programas de aprendizaje basados en simulación clínica aportan un entorno seguro para el alumno, y evitan que los errores se manifiesten en la atención a pacientes. El objetivo de este trabajo es compartir el protocolo usado en el briefing de actores para su entrenamiento, y caracterización fiel y estandarizada de sus roles como participantes simulados. El protocolo responde a la experiencia de aplicación de tres años (2016-2019) del grupo docente de investigación en simulación clínica de la Universidad Europea de Madrid. Contar con un protocolo funcional para entrenar participantes simulados que pueda ser utilizado para actores profesionales o no, eleva la fidelidad de los escenarios, facilita la organización, gestión y medición de los procesos de enseñanza, así como la gestión de recursos materiales y humanos, reduce costos, permite optimizar tiempos y favorece la evaluación y el mejor cumplimiento de los objetivos de aprendizaje del alumno.

\section{ABSTRACT}

Learning programmes based on clinical simulation provide a safe environment for the learner and prevent learning errors from manifesting themselves in patient care. The objective of this work is to share the protocol used in the briefing of actors for their training, faithful and standardized characterization of their roles as simulated participants. The protocol responds to the application experience of three years (2016-2019) of the research teaching group in clinical simulation of the Universidad Europea de Madrid. Having a functional protocol to train simulated participants that can be used to train professional actors or not, increases the fidelity of the scenarios, facilitates the organization, management and measurement of teaching processes, facilitates the management of material and human resources, reduces costs, allows optimizing times and favors the evaluation and better fulfillment of the student's learning objectives.

\section{INTRODUCCIÓN}

$\mathrm{E}^{\prime}$ empleo de actores en el aprendizaje basado en simulación clínica es una práctica cada vez más frecuente y diversa en la educación médica actual. Los términos "paciente estandarizado" y "paciente simulado" se utilizan a menudo para identificar a quien ha sido entrenado para representar a una persona real, que interactúa con un alumno en un escenario simulado y lo hace de forma realista y a veces estandarizada. ${ }^{1}$ Un paciente estandarizado es una persona que pretende ser un enfermo, un familiar e incluso un profesional de la salud. ${ }^{2}$

En sus normas de buenas prácticas, la Association of Standardized Patient Educators (ASPE) ${ }^{+}$ ofrece prioridad al término "participante simulado", como definición más inclusiva para referirse a todos los actores humanos que representan un determinado carácter y que pueden asumir papeles de paciente, familiar de paciente, profesional sanitario, entre otros.

El presente artículo acoge el mismo término de "participante simulado" (en adelante PS), atribuyéndole la categoría de personal entrenado para un fin escénico específico, y lo diferencia del término genérico de actor, al que identifica como la figura profesionalmente preparada, pero no entrenada en el rol sanitario o clínico específico.

En el concepto de PS incluimos al paciente simulado habitual, que hace de enfermo, y al confederado, entendido como aquel profesional de la salud, estudiante de otros niveles o especialidades, técnicos de simulación entrenados para ser "plantados" en un escenario para guiarlo. ${ }^{3}$ 
Para los desempeños de actuación simulada se suele contratar con frecuencia -y en dependencia de los presupuestos de las instituciones- a actores profesionales, considerando que tienen una formación dramatúrgica, y una capacidad entrenada en la reproducción que facilita el desempeño.

Las normas de buenas prácticas de la $\mathrm{ASPE}^{+}$ sientan una diferencia interesante entre un actor-persona que cumple los objetivos de un dramaturgo y/o director, y cuyo principal objetivo es entretener a un público -y un participante simulado, persona que realiza una actividad educativa, centrada en objetivos de aprendizaje y puesta al servicio de los alumnos. A lo que podríamos añadir que el PS debe interactuar con un docente de variable experiencia en términos de representación, que responde a intereses de un currículo y a una institución académica.

En la mayor parte de nuestras instituciones, los tiempos curriculares son escasos o muy limitados. Y existen pocos protocolos de gestión. De ahí que, con frecuencia, la capacitación que se ofrece corre el riesgo de convertirse en un briefing insuficiente para las múltiples habilidades que se le demandan al participante simulado., ${ }^{4}$

\section{Importancia del entrenamiento para la representación de roles simulados}

Sean o no actores profesionales, a los PS se les brinda una formación previa denominada coaching, briefing o capacitación, que la mayoría de las veces corre a cargo del experto que diseña el escenario o de sus colaboradores. Dicho entrenamiento procura elevar la calidad de las interpretaciones, garantizar su estandarización (consistencia, precisión) y repetibilidad. ${ }^{6}$ También se procura que cada PS responda a las necesidades individuales de los alumnos y los contextos, y esté a tono con los requerimientos de las escenas con la mayor autenticidad y flexibilidad posibles ${ }^{+}{ }^{1,7}$

Para el logro de esta espontaneidad o libre albedrío "controlado" del PS, también es necesaria la formación.

Otro factor importante que aporta la adecuada preparación de actores, es el crecimiento del nivel de verosimilitud y realismo de las escenas. La fidelidad absoluta en simulación clínica es, a veces, alcanzable cuando un actor estandarizado proporciona una historia realista y precisa, al pun-

\section{Tabla 1: Dinámicas de intercambio con el PS que un capacitador debe tener en cuenta.}

\begin{tabular}{|c|c|c|c|c|}
\hline $\begin{array}{l}\text { Tipo de } \\
\text { briefing }\end{array}$ & Sesiones & Detalles & Recomendaciones & $\begin{array}{l}\text { Tiempo } \\
\text { estimado }\end{array}$ \\
\hline \multirow[t]{4}{*}{$\begin{array}{l}\text { Briefing a } \\
\text { distancia }\end{array}$} & $\begin{array}{l}\text { 1. Selección } \\
\text { del PS }\end{array}$ & $\begin{array}{l}\text { De acuerdo con las características } \\
\text { físicas, etarias, étnicas y culturales } \\
\text { del rol }\end{array}$ & $\begin{array}{l}\text { - Tener un banco de PS } \\
\text { - Tener fichas con el background de perfiles de cada PS } \\
\text { - Seleccionar PS que sean precisos en la representación de perfiles similares } \\
\text { - Seleccionar PS, cuyos desempeños actorales previos, historias de vida o } \\
\text { memoria emocional guarden relación con el perfil a representar }\end{array}$ & $\begin{array}{l}20 \\
\text { minutos }\end{array}$ \\
\hline & $\begin{array}{l}\text { 2. Primer } \\
\text { contacto con } \\
\text { el PS }\end{array}$ & $\begin{array}{l}\text { Presentación de los objetivos } \\
\text { de la escena simulada } \\
\text { Envío de la ficha del PS } \\
\text { Se indica lectura de la ficha del PS }\end{array}$ & $\begin{array}{l}\text { Puede realizarse por vía telefónica o mediante aplicaciones de } \\
\text { mensajería de voz o escritas, previo consentimiento por parte de los } \\
\text { involucrados }\end{array}$ & $\begin{array}{l}10 \\
\text { minutos }\end{array}$ \\
\hline & $\begin{array}{l}\text { 3. Lectura de } \\
\text { la ficha por } \\
\text { parte del PS }\end{array}$ & $\begin{array}{l}\text { El PS realiza la lectura pormenori- } \\
\text { zada y reflexiva de la ficha }\end{array}$ & $\begin{array}{l}\text { - Se le indicará al PS recopilar las dudas para su referencia en la lectu- } \\
\text { ra conjunta con capacitador } \\
\text { - Se le indicará al PS la disponibilidad horaria del capacitador para } \\
\text { solventar las dudas urgentes }\end{array}$ & $\begin{array}{c}1 \\
\text { semana }\end{array}$ \\
\hline & $\begin{array}{l}\text { 4. Lectura } \\
\text { conjunta de } \\
\text { la ficha }\end{array}$ & $\begin{array}{l}\text { Capacitador y PS realizan la } \\
\text { lectura conjunta de la ficha }\end{array}$ & $\begin{array}{l}\text { La conversación puede ser grabada (podcast), previo consentimiento } \\
\text { de los involucrados para que quede como referencia cognitiva al PS, } \\
\text { capacitador, técnicos y otros capacitadores }\end{array}$ & $\begin{array}{l}30-45 \\
\text { minutos }\end{array}$ \\
\hline $\begin{array}{l}\text { Briefing } \\
\text { in situ }\end{array}$ & $\begin{array}{l}\text { 5. Orientación } \\
\text { al entorno }\end{array}$ & $\begin{array}{l}\text { El capacitador entrena al PS en } \\
\text { el uso del escenario }\end{array}$ & $\begin{array}{l}\text { - Capacitador y técnico de simulación, detallan las características } \\
\text { físicas del escenario, colocación de cámaras, recursos electrónicos y } \\
\text { demás elementos del atrezo que deban pormenorizar in situ }\end{array}$ & $\begin{array}{l}20 \\
\text { minutos }\end{array}$ \\
\hline
\end{tabular}

PS = paciente simulado. 


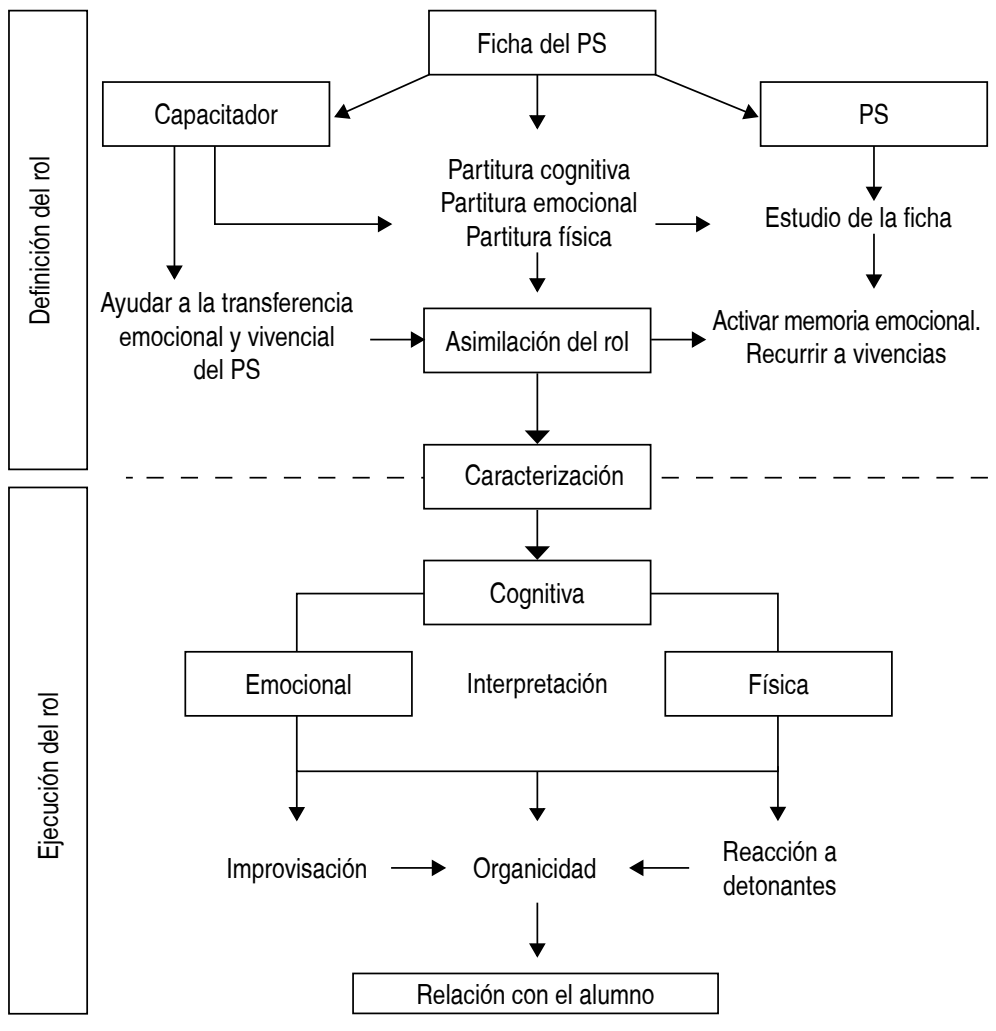

Figura 1: Estrategia de capacitación.

+ https://www.aspeducators.org/assets/docs/ASPE\%20SOBP\%20Infographic.pdf

to que ni siquiera un experto es capaz de detectar que se trata de una simulación. 8,9

La influencia del nivel de fidelidad de las simulaciones en el rendimiento de los estudiantes es un tema en discusión y análisis, ${ }^{10}$ pero la evidencia indica que con el adecuado entrenamiento de un participante simulado, se pueden lograr niveles de reproducibilidad del mundo real mucho mejores, y con menor costo que los que aportaría el uso de un simulador de alta fidelidad ingeniera. ${ }^{11,12}$

Por otro lado, los beneficios de retroalimentación y evaluación del estudiante que puede generar un actor entrenado son también aportes significativos, y se convierten en ayuda importante para alumnos y profesores. ${ }^{13-17}$

En este trabajo se presentan dinámicas de intercambio formativo, estrategias y resultados en la capacitación de PS, llevados a cabo en un periodo de tres años (2016-2019), por el grupo docente de investigación en simulación de la Universidad Europea.

Por su nivel de generalización y flexibilidad, estas estrategias pueden ser aplicadas en el entrenamiento de participantes simulados, sean o no actores profesionales, y se destinen tanto a la realización de escenarios de simulación clínica como a la Evaluación Clínica Objetiva y Estructurada (ECOE).

\section{DESARROLLO}

Capacitación de participantes simulados. La Tabla 1 refleja la dinámica de intercambio que un capacitador debe tener en cuenta para la formación de un PS, mediante estrategias escritas, orales y presenciales.

A su vez, se detallan las características de cada sesión, se plantean recomendaciones de cómo gestionarlas de manera más efectiva y se ofrece un estimado de tiempo para cada una. Lo que suma un total de hora y media de preparación conjunta, con una semana-como mínimo- para el estudio pormenorizado $y$, en determinados puntos, memorístico del rol por parte del PS.

En cuanto al factor tiempo, es útil aportar que estos estimados se reducen considerablemente cuando el capacitador conoce a profundidad el staff o banco de PS, con los cuales colabora o cuando los PS han interpretado casos similares.

Estrategia de capacitación. La Figura 1 muestra la estrategia de capacitación que se ha seguido. La Tabla 2 refleja la ficha que se utilizó para la capacitación de los PS. Es cumplimentada por el capacitador, en cada uno de sus apartados. Debe ser estudiada por el PS en un tiempo no inferior a una semana, y constituye el documento guía del proceder en la escena.

Tiene estructura de fácil lectura, pormenorizada en acápites y subacápites, lo que permite una comprensión fácil y rápida.

\section{CONCLUSIONES}

La posibilidad de tener un protocolo sólido, validado y funcional para entrenar PS, que pueda ser extensible al entrenamiento de actores, profesionales o no, permite la consolidación de iniciativas activas de aprendizaje que están validadas por la evidencia científica.

Tener una guía de entrenamiento para el paciente estandarizado, además de estructurar el trabajo de capacitación del PS, eleva la fidelidad de los escenarios, facilita la organización, gestión y medición de los procesos de enseñanza, reduce costos, permite optimizar tiempos, facilita la gestión de recursos materiales y humanos y favorece la evaluación y el mejor cumplimiento de los objetivos de aprendizaje del alumno. 


\section{Partitura del participante simulado}

\section{Partitura cognitiva: ¿Qué debe saber el PS? \\ Elementos \\ 1. Información general}

Información

del caso

Partitura emocional: ¿Qué debe transmitir emocionalmente el PS?

\section{Cómo se cumplimentan}

Suele corresponderse con el diagnóstico u objetivo principal de la escena Principales metas de aprendizaje que el alumno debe obtener, concretas y sencillamente enunciadas

Suele identificarse con el rol

Número de personas que intervendrán en la escena e interactuarán con el PS y sus roles

Estimado, en minutos, de lo que debe durar la escena

Información general imprescindible que el PS no puede dejar de conocer (En qué momento comienza la escena y en qué se basa)

Problema cognitivo principal, que plantea la escena y que el PS debe facilitar que se exprese por parte del alumno. (Debe corresponderse con el objetivo principal y estar en consonancia con el título de la escena)

Definición breve de los términos médicos relevantes a dominar por el PS

Conjunto de síntomas que el PS debe expresar de modo verbal o no verbal Nombre de los medicamentos que debe aportar el actor, así como sus dosis De ser necesario, se abundará en 1.12 y 1.15

Pruebas complementarias que debe aportar o referir verbalmente

Cifras numéricas que debe memorizar

Datos o conocimiento previo que debe tener el PS. (Historia médica, social o económica del rol, historia de antecedentes familiares o personales. Si es necesario, se indicarán lecturas, visualizaciones de vídeos, etcétera)

Información que debe tener cuidado de no aportar el PS

Información concreta y condiciones en que debe hacerlo

Elementos que debe aportar de modo preciso y que necesitará memorizar Información concreta y condiciones en que debe hacerlo

Información concreta y condiciones en que debe hacerlo

Acción, frase, decisión o indicador cualitativo o cuantitativo (catalizadores o inciting event que imponen un cambio en la gestión clínica) ante los que el actor debe reaccionar de una determinada manera

Definir el nivel de improvisación que puede tener y qué recurso verbal utilizar, en caso de que no recuerde

\section{Elementos}

Memoria emotiva previa

Gestión emocional de la escena

\section{Factores}

2.1 Historia emotiva del rol

2.2 Características emocionales básicas del rol

2.3 Información emocional clave

2.4 Desempeño emocional en las diferentes fases de la escena y cómo evidenciará su estado emocional de acuerdo con detonantes

\section{¿Cómo se cumplimentan?}

Antecedentes emocionales de peso y factores emocionales previos que tengan vínculo con la escena

Estado emocional global que manifestará durante la escena (contenido, indiferente, exaltado)

Información emocional imprescindible que el actor no puede dejar de conocer y representar

Rasgos visibles y audibles que expresen la emotividad de acuerdo con el momento y la caracterización emocional del actor, mediante recursos verbales y no verbales 
Continúa la Tabla 2: Ficha del PS.

\section{Partitura del participante simulado}

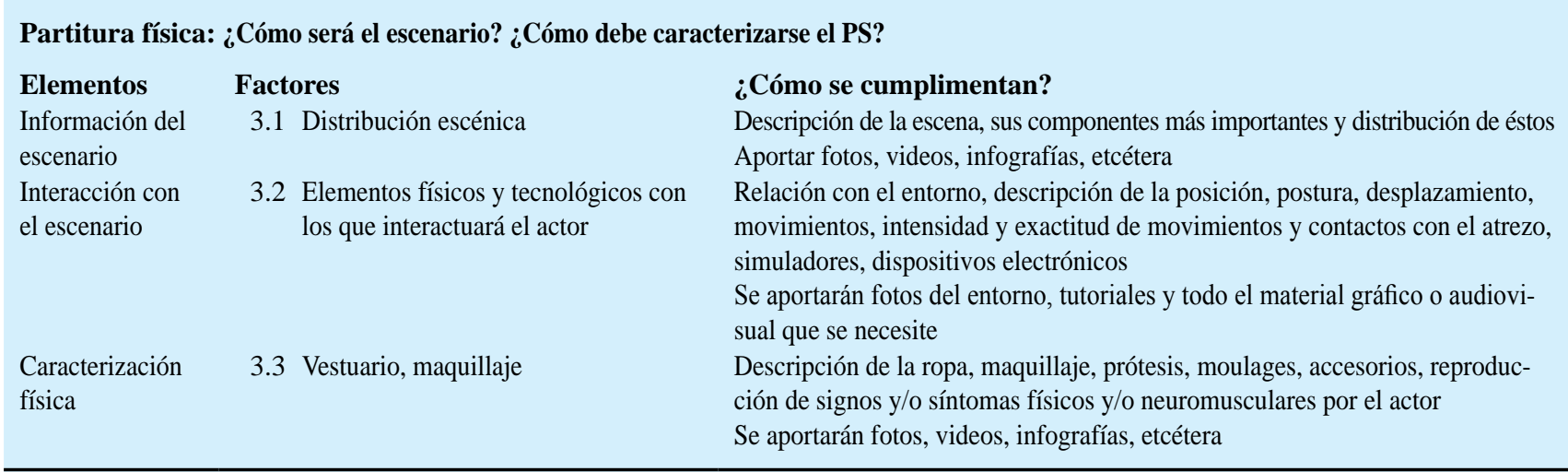

PS = paciente simulado.

\section{AGRADECIMIENTOS}

A los actores que se desempeñan en los ejercicios de simulación clínica.

\section{REFERENCIAS}

1. Lewis KL, Bohnert CA, Gammon WL, Hölzer H, Lyman L, Smith C, et al. The association of standardized patient educators (ASPE) standards of best practice (SOBP). Adv Simul. 2017; 2 (1): 10.

2. Dudley F, Silverman J. The simulated patient handbook: a comprehensive guide for facilitators and simulated patients. 4ed. Boca Raton: Chapman and Hall/CRC. 2018.

3. Wallace P. Coaching standardized patients: for use in the assessment of clinical competence. Springer Publishing Company. New York: Springer Publishing Company; 2006.

4. Heine N, Ferguson D. Management of Standardized Patient Programs. In: Palaganas JC, Maxworthy JC, Epps CA, Mancini MEB, editors. Defining Excellence in Simulation Programs. 1st ed. Philadelphia, PA: Lippincott Williams \& Wilkins; 2015. pp. 391-422.

5. Newlin-Canzone ET, Scerbo MW, Gliva-McConvey G, Wallace AM. The cognitive demands of standardized patients: Understanding limitations in attention and working memory with the decoding of nonverbal behavior during improvisations. Simul Healthc. 2013; 8 (4): 207-214. doi: 10.1097/SIH.0b013e31828b419e.

6. Wallace P. Following the threads of an innovation: the history of standardized patients in medical education. Caduceus. 1997; 13 (2): 5-28.

7. Williams B, Song JJY. Are simulated patients effective in facilitating development of clinical competence for healthcare students? A scoping review. Adv Simul. 2016; 1 (1): 6. doi: 10.1186/s41077-016-0006-1.

8. Tun JK, Alinier G, Tang J, Kneebone RL. Redefining simulation fidelity for healthcare education. Simul Gaming. 2015; 46 (2): 159-174.
9. Rethans JJ, Gorter S, Bokken L, Morrison L. Unannounced standardised patients in real practice: A systematic literature review. Med Educ. 2007; 41 (6): 537-549. doi: 10.1111/j.1365-2929.2006.02689.x.

10. Issenberg SB, Mcgaghie WC, Petrusa ER, Gordon DL, Scalese RJ. Features and uses of high-fidelity medical simulations that lead to effective learning: a BEME systematic review. Med Teach. 2005; 27 (1): 10-28.

11. Pascucci RC, Weinstock PH, O'Connor BE, Fancy KM, Meyer EC. Integrating actors into a simulation program: a primer. Simul Healthc. 2014; 9 (2): 120-126. doi: 10.1097/SIH.0b013e3182a3ded7.

12. Cleland J, Abe K, Rethans JJ. The use of simulated patients in medical education: AMEE Guide No. 42. Med Teach. 2009; 31 (6): 477-486.

13. Glassman PA, Luck J, O'Gara EM, Peabody JW. Using standardized patients to measure quality: evidence from the literature and a prospective study. Jt Comm J Qual Improv. 2000; 26 (11): 644-653.

14. Van der Vleuten CP, Swanson DB. Assessment of clinical skills with standardized patients: state of the art. Teach Learn Medic: An Int J. 1990; 2 (2): 58-76.

15. Madan AK, Caruso BA, Lopes JE, Gracely EJ. Comparison of simulated patient and didactic methods of teaching HIV risk assessment to medical residents. Am J Prev Med. 1998; 15 (2): 114-119.

16. Barrows HS. Training Standardized Patients to have physical findings. Chicago: Southern Illinois University School of Medicine. 1999.

17. Collins JP, Harden RM. AMEE Medical Education Guide No. 13: real patients, simulated patients and simulators in clinical examinations. Med Teach. 1998; 20 (6): 508-521.

Correspondencia:

Gleyvis Coro-Montanet

E-mail: gleyvis.coro@universidadeuropea.es 